\title{
Short-Pulse and Directional Thalamic Deep Brain Stimulation - Differential Effects in Parkinsonian and Essential Tremor
}

\author{
Ute Hidding \\ University Medical Center Hamburg-Eppendorf \\ Miriam Schaper \\ University Medical Center Hamburg-Eppendorf \\ Alessandro Gulberti \\ University Medical Center Hamburg-Eppendorf \\ Carsten Buhmann \\ University Medical Center Hamburg-Eppendorf \\ Christian Gerloff \\ University Medical Center Hamburg-Eppendorf \\ Christian Moll \\ University Medical Center Hamburg-Eppendorf \\ Wolfgang Hamel \\ University Medical Center Hamburg-Eppendorf \\ Chi-un Choe \\ University Medical Center Hamburg-Eppendorf \\ Monika Pötter-Nerger ( $\nabla$ m.poetter-nerger@uke.de) \\ University Medical Center Hamburg-Eppendorf
}

\section{Research Article}

Keywords: Parkinson's disease, Essential tremor, deep brain stimulation, directional stimulation, short pulse stimulation

Posted Date: May 28th, 2021

DOI: https://doi.org/10.21203/rs.3.rs-486187/v1

License: (9) This work is licensed under a Creative Commons Attribution 4.0 International License. Read Full License 


\section{Abstract}

The aim of this study was to assess the effects of novel stimulation algorithms of deep brain stimulation (short pulse and directional stimulation) in the ventrointermediate thalamus and posterior subthalamic area (VIM/PSA-DBS) on tremor in Parkinson's disease (PD) and to compare the effects with those in Essential tremor (ET). Methods: We recruited six PD patients (70.8 \pm 10.4 years) and seven ET patients $(64.4 \pm 9.9$ years) with implanted VIM/PSA-DBS in a stable treatment condition (>3months postoperatively). Tremor severity and ataxia were assessed in four different stimulation conditions in a randomized order: DBS switched off



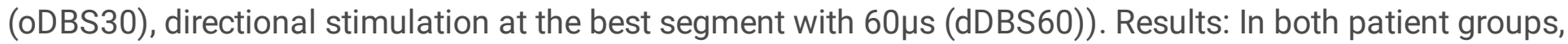
all three DBS stimulation modes reduced the total tremor score compared to STIM OFF, whereas stimulationinduced ataxia was reduced by oDBS30 and partially by dDBS60 compared to oDBS60. Tremor reduction was more pronounced in PD than in ET due to a limited DBS effect on intention and action-specific drawing tremor. Conclusion: In PD and ET tremor, the use of short pulse or directional VIM/PSA-DBS is an effective tool to enhance the therapeutic window.

The study was registered in the DRKS (ID DRKS00025329, 18.05.2021).

\section{Introduction}

Parkinsonian (PD) and Essential tremor (ET) are beyond the most common tremor syndromes [1-3] with considerable impact on patients' quality of life. In both disease conditions, deep brain stimulation (DBS) in the area of the ventral intermediate nucleus (VIM) and the posterior subthalamic area (PSA) is a good treatment option in case of drug therapy failure $[4,5]$. While the subthalamic nucleus (STN) or the globus pallidus internus (GPi) are often targeted in PD due to additional effects on bradykinesia and rigidity, VIM/PSA-DBS is preferred in elderly PD patients with lateralized tremor. VIM/PSA-DBS proved an effective treatment to suppress tremor, but its efficacy is limited by stimulation induced adverse effects [6-8]. In recent years, new forms of stimulation have expanded the therapeutic options. On the one hand, stimulation with short pulses $(<60 \mu \mathrm{s})$ is assumed to target specific nerve fibers along their different neurophysiological properties. On the other hand, a spatially restricted, more focal, directed electric field can be applied via segmented electrodes. For both stimulation modes, it has been recently demonstrated that the therapeutic window can be enhanced in ET patients with VIM/PSA-DBS [9-12] or PD patients with STN-DBS [13, 14]. To date, the use of novel stimulation algorithms in PD patients with VIM/PSA-DBS has not been assessed.

The pathophysiology of Parkinsonian and Essential tremor is quite different $[15,16]$. Parkinsonian tremor might be elicited by the combination of nigrostriatal degeneration and cerebello-thalamic circuit dysfunction. FDG-PET and functional MRI studies suggest that in PD activity changes in the basal ganglia network involving the putamen and globus pallidus are associated to tremor onset while cerebellum, thalamus and the motor cortex correlate with tremor amplitude [16], thus it was proposed that the basal ganglia circuit might trigger the cerebello-thalamo-cortical network to oscillate, resulting in tremor. In contrast, in ET the abnormality seems to be within the cerebello-thalamo-cortical network itself with a dysfunctional motor controller which sets off the oscillation [16]. The ventral intermediate nucleus (VIM) is a main relay nucleus 
embedded in the cerebello-thalamo-cortical circuit and an optimal target to interfere with circuit oscillations by DBS.

It is of interest, whether the effects of VIM/PSA-DBS depend on the preexisting pathophysiological state of the cerebello-thalamo-cortical circuit in these two disease conditions and whether the use of short pulse and directional stimulation can optimize potential effects and side effects in both tremor entities, Parkinsonian and Essential tremor. Thus, we aimed to evaluate the effect of short pulse and directional DBS in the VIM / PSA in PD patients and to compare DBS effects with those in ET patients.

\section{Materials And Methods}

\subsection{Study design:}

Six patients with tremor-dominant PD (all male) and seven ET-patients (six male) were included in this study. All but one PD patients were treated with unilateral thalamic DBS contralateral to the more affected side, all ET patients with bilateral thalamic DBS. Data of the ET patients have been published before [10]. One of the patients included in the former study was excluded from actual comparison because of newly diagnosed PD. For clinical details see Table 1. Written informed consent was obtained from all participants. The study protocol was approved by the Ethics Committee of the Hamburg Board of Physicians (PV5281). The assessments were conducted in accordance with the Declaration of Helsinki. The study was registered in the DRKS (ID DRKS00025329, 18.05.2021).

\subsection{Surgical procedure and definition of electrode position.}

All patients were implanted with the Infinity DBS system (St. Jude Medical Neuromodulation Division, Plano, TX, USA) according to standard surgical procedures at our center as previously described in detail [17]. Based on preoperative T1 magnetic resonance imaging and post-operative computed tomography scans, the $2^{\text {nd }}$ lowest contacts were located in the PSA. The optimal target site for electrode implantation was determined by multitrajectory microelectrode recordings (BenGun, up to three microelectrodes) and clinical evaluation of macrostimulation effects as described previously [18]. Coordinates of the electrode contacts were determined from the artifact in postoperative CT scans (Brainlab, Munich, Germany) in relation to the mid-commissural plane ( $x=$ medio-lateral, $y=$ anterior-posterior, $z=$ ventro-dorsal to mid-commissural point).

\subsection{Stimulation parameters}

As ataxia occurs mainly by ventral stimulation at distal contacts, patients were assessed at the lowest and 2nd lowest ventral contacts in the following four stimulation conditions: (1) no stimulation (STIM OFF) (2) omnidirectional suprathreshold stimulation with $60 \mu$ s and clinical signs of dysmetria and ataxia (3) omnidirectional stimulation with the same energy dose equivalent amplitude at constant frequency but applied with $30 \mu \mathrm{s}$ (4) directional stimulation with $60 \mu \mathrm{s}$. The required stimulation amplitude was determined by incremental amplitude increases of $0.5 \mathrm{~mA}$ with a pulse width of $60 \mu \mathrm{s}$ until the onset of ataxia and subsequent reduction in $0.1 \mathrm{~mA}$ steps as described earlier [19]. Suprathreshold stimulation was defined as stimulation amplitude $0.5 \mathrm{~mA}$ above the threshold for inducing ataxia. The total energy delivered (TEED) 


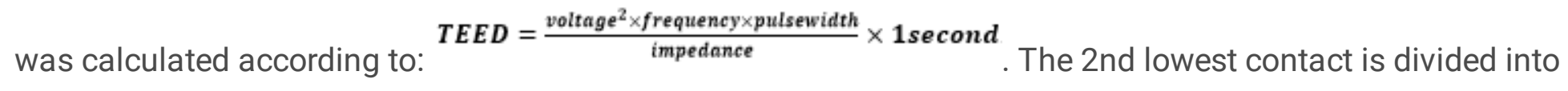
three single segments. To investigate effects of directional stimulation, the individual segments were

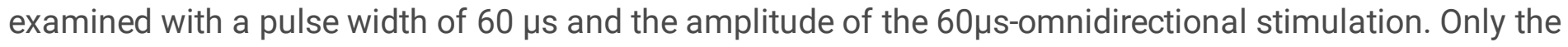
best segment was included in the analysis which was defined as the segment with the best effect on tremor or - in case of equal tremor effect - with the least induction of ataxia. Electrode contacts without ataxia side effects were excluded from the analysis (2 of 14 in PD, 2 of 28 in ET).

\subsection{Clinical assessment}

Severity of limb tremor was evaluated using the Fahn-Tolosa-Marin Tremor Rating Scale (FTMRS) using items 5 or 6 (rest, postural and intention tremor of right or left upper extremity), 11 (drawing large Archimedes spiral), 12 (drawing small Archimedes spiral) and 13 (drawing straight lines). FTMRS composite score was calculated by summing up items 5/6, 11, 12 and 13 (maximum score 24 with higher scores indicating more tremor). Severity of limb ataxia was evaluated using item 10 and 14 of the International Cooperative Ataxia Rating Scale (ICARS). ICARS composite score was calculated by summing up items 10 and 14 (maximum score 8 with higher scores indicating more severe dysmetria and ataxia). Paresthesia were evaluated using a visual analog scale ( 0 - none, 10 - strongest sensation). For comparison with preoperatively collected UPDRS data, a sum score from the tremor items for the upper extremity of the MDSUPDRS III was also calculated in PD patients (item 3.15 - 3.18). In ET patients, the FTMRS was collected preoperatively. Patients and examiners were blinded for the stimulation condition which were randomized by a second investigator.

\subsection{Statistical analysis}

All data are given as mean \pm standard deviation (SD). In a first step, PD patients were analyzed as a separate group. GLM ANOVA with the intrasubject factor stimulation condition (pulse width or directional/omnidirectional stimulation) was performed for the main symptom domains tremor, ataxia and paresthesia. In a second step, GLM ANOVA with the intrasubject factor stimulation condition (pulse width or directional/omnidirectional stimulation) and the intersubject factor disease condition (PD vs. ET) was performed. Greenhouse Geisser correction was used, if sphericity was violated. Post-hoc 2-tailed paired ttests were used to specify intrasubject effects and unpaired t-tests to compare group effects. Statistical analyses were performed with SPSS (IBM SPSS Statistics for Windows, version 20, SPSS Inc., Chicago, IL, USA) and GraphPad Prism (version 9 for Windows, La Jolla, USA).

\section{Results}

\subsection{Subjects characteristics}

Detailed group characteristics are summarized in Table 2. ET and PD - groups did not differ in terms of age or time since surgery. Mean age was $70.8 \pm 10.4$ years (PD patients) and $64.4 \pm 9.9$ years (ET patients, $p=0.28$ ), mean duration of DBS treatment was 174.8 \pm 102.7 days (PD patients) and $162.6 \pm 52.6$ days (ET patients, $p=0.79$ ). ET patients showed a significant longer disease duration (PD: 6.8 \pm 3.1 years; ET: $33.6 \pm 18.7$ years; 
$p=0.002)$. The position of the electrodes did not differ significantly between PD and ET patients. Given the border between VIM and PSA at the level of the midcommissural line, all but one contact investigated were located in the PSA. The stimulation amplitude to induce ataxia tended to be higher in PD patients but the difference was not significant (PD 3.58 $\pm 0.68 \mathrm{~mA}$. ET 3.19 $\pm 0.53 \mathrm{~mA}, \mathrm{p}=0.06$ ).

\subsection{Perioperative tremor scores in PD and ET patients}

The course of pre- and postoperative tremor scores in the observation period of about 6 months differed between both patient groups (Fig. 1). In PD patients, there was a slight, but not significant dopaminergic tremor response in a preoperative L-Dopa challenge using suprathreshold morning dosage (UPDRS-tremor score: preoperatively MedOFF 10.4 \pm 2.2 , MedON 8.6 \pm 2.4 ). The pre- and postoperative STIM OFF tremor scores at the time of the experiment were comparable (UPDRS III tremor score: pre-DBS Med OFF 10.4 \pm 2.2 , post-DBS STIM OFF 10.14 $\pm 1.35, p=0.10$ ) (Fig. 1A) indicating stable tremor characteristics in the off condition in the observation period. In contrast, tremor scores of ET patients significantly worsened in the longitudinal time course with significant perioperative increase of tremor severity (FTMRS sum score: preDBS 12.79 \pm 4.49 , post-DBS $15.79 \pm 3.58(p=0.005$; Fig. 1B). Noteworthy, the interval between pre- and postoperative assessments did not differ in PD and ET patients (Table 2).

\subsection{Conventional and new stimulation modes of VIM/PSA-DBS in PD patients}

In PD patients, new stimulation forms as short pulse stimulation and directional stimulation were compared to conventional omnidirectional $60 \mu \mathrm{s}$ VIM/PSA-DBS at two different contact levels, the most ventral ring contact and the adjacent dorsal, segmented contact (Fig. 2A).

The use of short pulse stimulation was equivalent in suppressing tremor compared to conventional VIM/PSA-DBS, but with less side effects at the ventral contact. GLM ANOVA with the factor stimulation mode (3 levels: 1. STIM OFF 2. oDBS60 3. oDBS30) revealed significant effects for the total tremor score $(F=36.66, p=0.001)$ and ataxia $(F=11.93, p=0.003)$. Paresthesia were not significantly different during the different STIM conditions $(F=3.08, p=0.11)$.

The total tremor score was significantly improved by both oDBS60 $(2.14 \pm 0.9 ; p=0.001)$ and oDBS30 $(1.71 \pm 0.76, p=0.001)$ compared to OFF $(12.86 \pm 4.6)$, there was no significant difference between both stimulation modes indicating comparable tremor improvement by both stimulation modes. Interestingly, the effect of DBS was divergent on the different tremor subtypes as tremor at rest, postural tremor, intention tremor and tremor while drawing Archimedes spiral (Fig 3A). A two factorial GLM ANOVA with the intrasubject factor 1. STIM condition and 2. tremor subtype revealed a highly significant effect for STIM $(F=84.46, p<0.001)$ and tremor subtype $(F=22.97, p<0.001)$ with significant interaction $(F=12.63, p=0.001)$. Both stimulation modes improved significantly tremor at rest (oDBS60 and oDBS30 $p<0.001$ ), postural tremor (oDBS60 and oDBS30 $p<0.001$ ) and slightly tremor while drawing spirals (oDBS60 $p=0.022, \mathrm{oDBS} 30$ $p=0.021$ ) compared to STIM OFF, but not sufficiently intention tremor (oDBS60 and oDBS30 p $=0.078$ ). Thus, both stimulation modes improved tremor compared to STIM OFF, however with a restricted effect on intentional tremor - this pattern of specific tremor subtype suppression was similar between conventional or short-pulse stimulation. 
Both stimulation modes differed in terms of side effects. The use of conventional oDBS60 (3.0 \pm 1.0$)$ induced significant ataxia, which was not present in STIM OFF. Stimulation-induced ataxia was significantly less accentuated while oDBS30 $(1.71 \pm 0.76)$ compared to oDBS60 $(p=0.021)$ indicating a better side effect profile using short pulses (Fig 2B).

The use of directional stimulation revealed a similar pattern when comparing omnidirectional and directional stimulation at the adjacent dorsal contact using the same pulse width of $60 \mu \mathrm{s}$. The total tremor score was significantly reduced (GLM ANOVA factor STIM condition $F=29.41, p=0.003$ ) by both stimulation forms (oDBS60 3.0 $\pm 0.7, p=0.001$; dDBS60 1.34 $\pm 0.6, p=0.006)$ compared to STIM OFF $(12.86 \pm 4.6)$ with no significant differences between both stimulation modes. Again, there was a tremor subtype specific effect of stimulation (GLM ANOVA factor STIM condition $F=66.99, p=0.001$, tremor subtype $F=14.27, p=0.01$ ) and the interaction $(F=7.76, p=0.012$; Fig $3 A)$. The pattern of tremor improvement was similar between both stimulation modes. Directional stimulation at the best segment reduced tremor at rest $(p=0.001)$, postural tremor $(p=0.004)$ but there was no significant improvement of intentional or drawing tremor.

In terms of the side effect ataxia (GLM ANOVA STIM F=22.34, $p=0.007$ ), directional stimulation at the best segment (dDBS60 2.40 \pm 0.55 ) tended to induce less ataxia than omnidirectional DBS (oDBS60: $3.40 \pm 1.14$ ), but the difference was not significant between both stimulation modes.

In summary, VIM/PSA-DBS, regardless of the stimulation mode, improved resting and postural tremor, but not intention tremor and slightly less drawing tremor in PD patients. Short-pulse and directional DBS were comparably efficient in the improvement of tremor compared to conventional DBS, but with less side effects as ataxia, particularly with short-pulse stimulation.

\subsection{Comparison of VIM/PSA-DBS effects between PD and ET patients}

\subsubsection{Comparison of DBS effects with short pulse stimulation}

The total FMTRS was significantly reduced by VIM/PSA-DBS at the ventral contact in both patient groups regardless of the pulse width. GLM ANOVA with the intrasubject factor stimulation condition (3 levels: 1.


2. ET) revealed a highly significant difference for the factor stimulation condition $(F=113.31, p<0.001)$, but no significant interaction between stimulation condition and disease $(F=0.17, p=0.76)$. Comparable to PD patients, the total tremor score was significantly reduced in ET patients in both ON-condition (OFF:

$15.79 \pm 3.58$, oDBS60: $6.00 \pm 3.16$, oDBS30 5.0 \pm 3.01 , both $p<0.0001$ ) with no significant differences between STIM ON conditions.

However, the DBS effects were diverging in respect of disease condition and the effect on the different tremor entities as tremor at rest, postural tremor, intention tremor and tremor while drawing Archimedes spiral (Fig 3). A two-factorial GLM ANOVA with the intrasubject factor STIM condition and tremor subtype revealed a highly significant effect again for STIM $(F=164.04, p<0.001)$ and tremor subtype $(F=41.44$, $p<0.001)$, with significant interaction of tremor subtype, disease condition $(F=34.05, p<0.001)$ and STIM condition $(F=27.77, p<0.001)$ indicating a disease specific action of short pulse and conventional DBS on 
the different tremor subtypes. This finding could be replicated testing short pulse and conventional DBS at the more dorsal contact (STIM $(F=67.75, p<0.001)$, revealing significant effects for tremor subtype $(F=45.7$, $p<0.001)$ with significant interaction with disease condition $(F=20.16, p<0.001)$ and with both, disease and STIM condition $(F=13.97, p<0.001)$. In detail, the predominant tremor at rest and postural tremor in PD patients was completely suppressed by VIM/PSA-DBS, whereas intentional and action tremor, particularly drawing spirals, was less impacted by VIM/PSA-DBS, which was more prominent in ET patients.

When normalising the total tremor scores in STIM ON in relation to the STIM OFF condition with lower percentages indicating better tremor reduction, we observed differential effects of DBS in both patients groups. The relative tremor reduction was more pronounced in PD patients for both stimulation modes compared to ET. This disease-specific effect was significant for oDBS60 and oDBS30 (oDBS60: PD 19.66\% \pm 7.2 , ET 40.46\% $\pm 22.5, p=0.0003$; oDBS30: PD 15.86\% \pm 10.63 , ET 31.18\% $\pm 22.64, p=0.0024$ (Fig 4A).

Suprathreshold stimulation with $60 \mu$ s at the ventral contact triggered ataxia as measured by the ICARS sum score (PD: OFF: $0.38 \pm 0.37$, oDBS60: $2.86 \pm 1.07$ p<0.0001; ET: OFF: $0.14 \pm 0.36$, oDBS60: $2.86 \pm 1.35$ $\mathrm{p}<0.0001$ ). GLM ANOVA with the intrasubject factor stimulation condition (3 levels: 1. STIM OFF 2. STIM ON

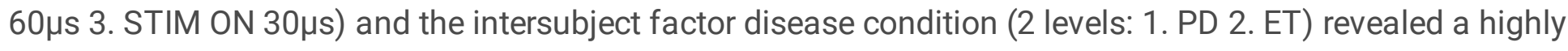
significant effect for the factor stimulation condition ( $F=36.86, p<0.001)$, but no significant interaction between stimulation condition and disease $(F=1.37, p=0.27)$. Post-hoc analyses revealed that ataxia induced by conventional $60 \mu \mathrm{s}$ DBS could be significantly reduced by usage of short-pulse stimulation with $30 \mu \mathrm{s}$ in both patient groups (PD: $1.43 \pm 0.78 p=0.0001, E T: 2.29 \pm 0.64 p=0.0001$ ) (Fig 1B). The same could be observed for stimulation effects at the second most dorsal contact (stimulation condition $(F=33.58, p<$ $0.001)$, no significant interaction with disease condition ( $F=0.62, p=0.52)$.

Again, we compared disease conditions more in detail by calculating the relative difference between the ICARS sum score with oDBS60 and the new stimulation condition (DeltalCARS), with larger values indicating less ataxia with the new stimulation condition. The use of oDBS30 resulted in better ataxia reduction in PD patients compared to ET patients (PD: 1.83 \pm 1.11 , ET 0.92 \pm 1.32 p=0.040; Fig 4B).

Paresthesia were assessed in both patient groups while application of the different stimulation modes. GLM ANOVA revealed a significant effect for the STIM condition at the ventral $(F=7.99, p=0.006)$ and dorsal contact $(F=8.53, p=0.003)$, but no significant interaction with disease condition indicating similar effects in both patient groups

\subsubsection{Comparison of omnidirectional and directional stimulation in PD and ET patients}

To evaluate and compare the effect of omnidirectional and segmented VIM/PSA-DBS in both patients groups, the stimulation effect with $60 \mu$ s in the ring mode and at the best segment was compared for the total tremor score, the tremor subtypes and the potential side effects ataxia and paresthesia.

The total tremor score was improved by both stimulation modes compared to STIM OFF condition in both patient groups. GLM ANOVA revealed a significant effect for stimulation condition $(F=60.442, p<0.001)$, but no significant interaction with disease condition. The different tremor subtypes were differentially impacted 
by DBS. There was a significant effect for the factor tremor subtype $(F=39.71, p<0.001)$ with significant interaction with disease condition $(F=19.9, p<0.001)$ as well as with stimulation mode and disease condition $(F=14.25, p<0.001)$ revealing tremor-specific action of the different stimulation modes in both disease entities. Again, the predominant resting tremor in PD patients was improved by omni- and unidirectional stimulation. In ET patients, the postural and intention tremor was improved, but the predominant drawing tremor was less impacted by both stimulation modes.

Comparing relative changes of DBS compared to STIM OFF with lower values indicating better symptom reduction, the relative reduction of tremor was comparable in PD patients compared to ET (dDBS60: PD $19.42 \% \pm 12.26$, ET $38.44 \% \pm 31.29, p=0.12$; Fig $4 A$ ). In terms of the reduction of stimulation induced ataxia, the use of dDBS60 showed no significant difference between patient groups (DeltalCARS: dDBS60: PD $0.80 \pm 1.5$, ET $1.86 \pm 0.86, p=0.11$; Fig 4B).

\section{Discussion}

The aim of this study was to assess the effects of new stimulation algorithms of VIM/PSA-DBS on tremor in PD patients and compare the effects with those in ET patients. The different tremor characteristics of the two patient groups have to be considered in the interpretation of VIM/PSA-DBS effects. Within the observation period of about 6 months, there was no natural progression of tremor severity in PD patients, but in ET patients in the STIM OFF condition. Tremor occurrence in advanced PD patients was accentuated at rest, but also emerged as a slight postural, intentional and task-specific tremor at drawing of Archimedes spirals. In contrast, in ET patients we observed no tremor at rest, but postural, intention and particularly taskspecific drawing tremor. Another main finding was the differential effect of VIM/PSA-DBS on the tremor subtypes. Whereas tremor at rest and postural tremor were improved sufficiently by new and conventional DBS stimulation modes, intentional or task-specific drawing tremors were less or not adequately improved by all tested stimulation modes of VIM/PSA-DBS. Since intentional and drawing tremor were predominant tremor features in ET, VIM/PSA-DBS seemed to improve less the total tremor score in ET than PD. This disease-specific effect with better tremor improvement in PD was significant for conventional and short pulse VIM/PSA-DBS, but not for directional DBS.

There are quite well-known effects of conventional VIM/PSA-DBS in PD and ET tremor. The conventional settings of VIM/PSA-DBS are usually a continuous delivery of pulses with a frequency of $130 \mathrm{~Hz}$ and $60 \mu \mathrm{s}$ pulse width. In a large European, multicenter study [4], upper and lower limb tremor scores were improved in both patient groups. Tremor at rest was reduced by $85 \%$ in parkinsonian patients and postural tremor was decreased in $89 \%$ the ET patients associated with improvement of activities of daily living in both groups [4]. Parkinsonian tremor has been treated in the last decades by DBS in various targets as globus pallidus, subthalamic nucleus, pedunculopontine nucleus or caudal zona incerta. Nevertheless, in a recent metanalysis the VIM has been proven to be the better target for Parkinsonian tremor reduction in the medication off condition [20]. A review of VIM-DBS effects in ET patients reported an improvement of tremor scores particularly of upper limb tremor by $90 \%$ [21]. Although conventional VIM-DBS is quite beneficial in the treatment of tremor, there are limitations of that therapeutical approach, particularly in terms of side 
effects as ataxia or paresthesia, which narrow the therapeutic window and require DBS reprogramming and trouble-shooting.

Particularly the induction of ataxia by stimulation of the ventral VIM or the adjacent PSA has recently become a focus of interest. It has been demonstrated that DBS of PSA is more effective in improving tremor than the VIM itself $[8,5]$. The anatomical background is that cerebello-thalamo-cortical fibers of the PSA are bundled together in a "bottle-neck", so that even low stimulation amplitudes excite all target fibers [8]. In contrast, ventral PSA stimulation induced more often unintentional side effects as ataxia, which was less pronounced while stimulating the more dorsal contacts [22, 23]. Typically, this syndrome manifests several months after an initially effective therapy and necessitates frequent adjustments in stimulation parameters [23]. In MR based studies the fibers involved in tremor suppression and induction of ataxia could be further characterized. The fibers arriving from the dentate nucleus of the contralateral cerebellum, travelling through the bottle-neck to the thalamus and the motor cortex seemed to be the target fibers for tremor reduction. In contrast, cerebello-rubro-spinal fibers might be possibly involved in the induction of ataxia by suprathreshold stimulation $[23,22]$. This close vicinity of fibers mediating beneficial effects for tremor suppression and fibers involved in pathogenesis of unintentional side effects, necessitates the use of specific DBS settings precisely targeting dentato-cerebello-thalamic fibres and preventing stimulation of cerebello-rubro-spinal fibers.

Recently, the introduction of new DBS devices enabled the release of short-pulse stimulation and the possibility to steer the current by directional stimulation through segmented leads.

Short pulse DBS within the STN revealed in PD patients an increased therapeutic window and a reduction of unintentional side effects such as dysarthria $[14,13,24,25]$. Besides, this new stimulation option was proven to be energy-saving compared to DBS with longer pulses and therefore minimized the frequency of recharging the DBS battery $[14,13,24,25,11]$. Furthermore, short-pulse VIM/PSA-DBS in ET patients was shown to be clinically advantageous with lower incidence of side effects as paresthesia, hand- and gait ataxia $[10,26]$. The effect of short-pulse and directional stimulation in the VIM/PSA on tremor and ataxia in PD patients was not assessed so far.

The potential mechanisms of action of short pulses DBS are currently investigated. From computational modelling it has been proposed that short-pulses might be more specific to activate selectively smalldiameter, local axons around the electrode tip compared to more distant and thick myelinated axons of the capsula interna along the chronaxie of neuronal elements, resulting in decreased side effects and an increased therapeutic window [13]. Another recent computational modelling study proposes that short pulses reduce side effects by non-dose equivalent stimulation resulting in reduced spread of neural activation [27]. While there are incongruent computational results in terms of mechanisms of action of short pulses, the clinical effect of reducing side effects due to unintentional co-stimulation of neighbor structures has become an important strategy of DBS troubleshooting in the clinical routine. With respect to the parkinsonian tremor, we hypothesized that short pulse DBS would enhance the dentate-cerebellar-thalamic fiber selectivity and preserve the accidental co-stimulation of neighbor rubro-cerebellar fibers, optimizing the 
therapeutic window. In fact, we found efficient tremor reduction by oDBS30 with significant reduction of stimulation induced ataxia resulting in an enhanced therapeutic window.

Directional DBS was tested in intraoperative and postoperative settings in PD and ET patients [28-31, 12]. The commercially available electrodes consist of leads with two middle rings divided into three segments. This electrode design enables the selection of only one segment resulting in axially defined asymmetric field restriction. Directional stimulation has therefore the potential to optimize spatially stimulation energy at the specific target fiber bundles, while preventing co-stimulation of fibers mediating unintentional side effects that may occur with omnidirectional stimulation. In previous studies using experimental lead designs during intraoperative recording $[29,28]$ and in postoperative studies implanting directional leads [30-32], the therapeutic window could be enhanced by directional stimulation compared to omnidirectional stimulation. In particular, the stimulation amplitude for symptom relief was reduced, while the threshold for the induction of side effects was incremented. The real-world differences between directional and omnidirectional STNDBS in PD patients were recently assessed in a large, prospective, international, multi-centre, double-blind randomized crossover study (ClinicalTrials.gov Identifier: NCT02989610).

In the present study, the use of directional DBS was equally efficient in the reduction of the total tremor score compared to omnidirectional stimulation, still, dDBS60 was slightly inferior in the improvement of specific tremor subtypes as drawing tremor in PD patients. The application of dDBS60 tended to reduce slightly stimulation-induced side effects as ataxia, however this effect was not significant. When comparing the use of directional DBS and short-pulse DBS, short-pulse DBS seemed to be slightly superior compared to dDBS60 in the widening of the therapeutic window, however one needs to consider the high amplitudes used in the study which might lower the spatial accuracy of dDBS60.

We found differential effects of the conventional and new stimulation modes between PD patients and ET patients in terms of tremor reduction and the extent of stimulation induced ataxia. The total tremor score seemed to be more improved in PD patients compared to ET patients by use of oDBS60 and oDBS30, though it must be considered that the tremor pattern was different between both patient groups. Besides, in PD patients the use of short pulses was even more effective in the reduction of stimulation-induced ataxia than in ET patients. These differential DBS effects might be explained by current pathophysiological assumptions of these different tremor entities in PD and ET patients. In PD, it is proposed that dysfunction within the basal ganglia-thalamo-cortical network could be the driver for the tremor onset, triggering then the cerebello-thalamic-cortical circuit which might be responsible for the tremor amplitude on its part [16]. In $\mathrm{fMRI}, \mathrm{PD}$ tremor onset was correlated with activity changes in the putamen and globus pallidus, while PD tremor amplitude was correlating with cerebellum, thalamus and cortex [33]. In FDG-PETs of PD patients, the amount of tremor amplitude could be also correlated with activity changes in the dentate nucleus, rostral parts of the cerebellum and motor cortex [34]. In contrast, in ET patients the main pathology might be located within the cerebello-thalamo-cortical loop with a dysfunctional motor controller in the cerebellum [16]. In ET patients, cerebellar pathology has been described [35, 36]. Thus it was proposed that the direct generator of both tremor entities might be the cerebello-thalamo-cortical network, however the difference might be the way, this network is activated to oscillate. 
Back to the findings of our study, one hypothesis could be that in PD patients the fiber bundles in this specific "bottle neck" within the cerebello-thalamo-cortical circuit are less affected by degenerative pathology than in ET, since in PD the main pathology is located in the basal ganglia. Therefore, DBS might interfere with an oscillating, but otherwise functioning network in PD. In ET, the pathological degeneration of cerebellar outflow fibers might cutback and limit DBS therapeutical effects, particularly in terms of preexisting ataxia due to cerebellar degeneration.

In conclusion, the application of new stimulation algorithms as short pulses or directional VIM/PSA-DBS might be beneficial in the tremor treatment of both PD and ET patients, particularly in the reduction of side effects as ataxia. In PD patients the usage of the new stimulation forms to enhance the therapeutic window seemed to be even more promising compared to ET, probably due to a different tremor phenomenology and pathophysiology.

\section{Declarations}

Funding: This research was funded by the Deutsche Forschungsgemeinschaft (DFG), SFB 936 (SFB 936/C8: Monika Pötter-Nerger \& Christian KE Moll)

Acknowledgments: We thank Marlies Schütte and Maja Kirsten for their contributions.

Author Contributions: UH and CUC conceived and designed the study. UH, MS and CUC acquired the data. UH and MP analyzed and interpreted the data. UH and MP drafted the manuscript. UH prepared the figures. MS and WH conducted all surgeries. All authors revised the manuscript for intellectual content.

Conflicts of Interest: CB has received speaking fees from Abbvie, Bial, TAD Pharma, UCB Pharma and Zambon and travel expenses from Medtronic. CG received fees as a consultant or lecture fees from Bayer Vital, Boehringer Ingelheim, EBS technologies, GlaxoSmithKline, Lundbeck, Pfizer, Sanofi Aventis, Silk Road Medical and UCB. CM has received fees for teaching, lectures and consultations from Abbott, lecture fees from Inomed and fees from Brainlab for software testing. WH has received honoraria for lectures, participation in advisory boards and travel expenses from Abbott, Boston Scientific and Medtronic. MP has received honoraria for lectures, participation in advisory boards and travel expenses from Boston Scientific, Abbott, Medtronic, Licher MT and Abbvie. UH, MS, AG and CC declare no commercial or financial relationships that could be construed as a potential conflict of interest.

\section{References}

1. Louis ED, Ferreira JJ (2010) How common is the most common adult movement disorder? Update on the worldwide prevalence of essential tremor. Mov Disord 25 (5):534-541. doi:10.1002/mds.22838

2. Chen W, Hopfner F, Becktepe JS, Deuschl G (2017) Rest tremor revisited: Parkinson's disease and other disorders. Transl Neurodegener 6:16. doi:10.1186/s40035-017-0086-4

3. Bhatia KP, Bain P, Bajaj N, Elble RJ, Hallett M, Louis ED, Raethjen J, Stamelou M, Testa CM, DeuschI G, Tremor Task Force of the International P, Movement Disorder S (2018) Consensus Statement on the 
classification of tremors. from the task force on tremor of the International Parkinson and Movement Disorder Society. Mov Disord 33 (1):75-87. doi:10.1002/mds.27121

4. Limousin P, Speelman JD, Gielen F, Janssens M (1999) Multicentre European study of thalamic stimulation in parkinsonian and essential tremor. J Neurol Neurosurg Psychiatry 66 (3):289-296

5. Barbe MT, Reker P, Hamacher S, Franklin J, Kraus D, Dembek TA, Becker J, Steffen JK, Allert N, Wirths J, Dafsari HS, Voges J, Fink GR, Visser-Vandewalle V, Timmermann L (2018) DBS of the PSA and the VIM in essential tremor: A randomized, double-blind, crossover trial. Neurology. doi:10.1212/WNL.0000000000005956

6. Cury RG, Fraix V, Castrioto A, Perez Fernandez MA, Krack P, Chabardes S, Seigneuret E, Alho EJL, Benabid AL, Moro E (2017) Thalamic deep brain stimulation for tremor in Parkinson disease, essential tremor, and dystonia. Neurology 89 (13):1416-1423. doi:10.1212/WNL.0000000000004295

7. Dick JP (2003) Multicentre European study of thalamic stimulation in essential tremor. J Neurol Neurosurg Psychiatry 74 (10):1362-1363

8. Herzog J, Hamel W, Wenzelburger R, Potter M, Pinsker MO, Bartussek J, Morsnowski A, Steigerwald F, Deuschl G, Volkmann J (2007) Kinematic analysis of thalamic versus subthalamic neurostimulation in postural and intention tremor. Brain 130 (Pt 6):1608-1625. doi:10.1093/brain/awm077

9. Bruno S, Nikolov P, Hartmann CJ, Trenado C, Slotty PJ, Vesper J, Schnitzler A, Groiss SJ (2020) Directional Deep Brain Stimulation of the Thalamic Ventral Intermediate Area for Essential Tremor Increases Therapeutic Window. Neuromodulation. doi:10.1111/ner.13234

10. Choe CU, Hidding U, Schaper M, Gulberti A, Koppen J, Buhmann C, Gerloff C, Moll CKE, Hamel W, PotterNerger M (2018) Thalamic short pulse stimulation diminishes adverse effects in essential tremor patients. Neurology. doi:10.1212/WNL.0000000000006033

11. Moldovan AS, Hartmann CJ, Trenado C, Meumertzheim N, Slotty PJ, Vesper J, Schnitzler A, Groiss SJ (2018) Less is more - Pulse width dependent therapeutic window in deep brain stimulation for essential tremor. Brain Stimul. doi:10.1016/j.brs.2018.04.019

12. Rebelo P, Green AL, Aziz TZ, Kent A, Schafer D, Venkatesan L, Cheeran B (2018) Thalamic Directional Deep Brain Stimulation for tremor: Spend less, get more. Brain Stimul. doi:10.1016/j.brs.2017.12.015

13. Reich MM, Steigerwald F, Sawalhe AD, Reese R, Gunalan K, Johannes S, Nickl R, Matthies C, Mclntyre CC, Volkmann J (2015) Short pulse width widens the therapeutic window of subthalamic neurostimulation. Ann Clin Transl Neurol 2 (4):427-432. doi:10.1002/acn3.168

14. Steigerwald F, Timmermann L, Kuhn A, Schnitzler A, Reich MM, Kirsch AD, Barbe MT, Visser-Vandewalle V, Hubl J, van Riesen C, Groiss SJ, Moldovan AS, Lin S, Carcieri S, Manola L, Volkmann J (2018) Pulse duration settings in subthalamic stimulation for Parkinson's disease. Mov Disord 33 (1):165-169. doi:10.1002/mds. 27238

15. Dirkx MF, Zach H, van Nuland A, Bloem BR, Toni I, Helmich RC (2019) Cerebral differences between dopamine-resistant and dopamine-responsive Parkinson's tremor. Brain 142 (10):3144-3157. doi:10.1093/brain/awz261

16. Hallett M (2014) Tremor: pathophysiology. Parkinsonism Relat Disord 20 Suppl 1:S118-122. doi:10.1016/S1353-8020(13)70029-4 
17. Buhmann C, Huckhagel T, Engel K, Gulberti A, Hidding U, Poetter-Nerger M, Goerendt I, Ludewig P, Braass H, Choe CU, Krajewski K, Oehlwein C, Mittmann K, Engel AK, Gerloff C, Westphal M, Koppen JA, Moll CKE, Hamel W (2017) Adverse events in deep brain stimulation: A retrospective long-term analysis of neurological, psychiatric and other occurrences. PLoS One 12 (7):e0178984.

doi:10.1371/journal.pone.0178984

18. Steigerwald F, Potter M, Herzog J, Pinsker M, Kopper F, Mehdorn H, Deuschl G, Volkmann J (2008) Neuronal activity of the human subthalamic nucleus in the parkinsonian and nonparkinsonian state. $\mathrm{J}$ Neurophysiol 100 (5):2515-2524. doi:10.1152/jn.90574.2008

19. Hidding U, Schaper M, Moll CKE, Gulberti A, Koppen J, Buhmann C, Gerloff C, Potter-Nerger M, Hamel W, Choe CU (2019) Mapping stimulation-induced beneficial and adverse effects in the subthalamic area of essential tremor patients. Parkinsonism Relat Disord 64:150-155. doi:10.1016/j.parkreldis.2019.03.028

20. Lin F, Wu D, Yu J, Weng H, Chen L, Meng F, Chen Y, Ye Q, Cai G (2021) Comparison of efficacy of deep brain stimulation and focused ultrasound in parkinsonian tremor: a systematic review and network meta-analysis. J Neurol Neurosurg Psychiatry. doi:10.1136/jnnp-2020-323656

21. Deuschl G, Raethjen J, Hellriegel H, Elble R (2011) Treatment of patients with essential tremor. Lancet Neurol 10 (2):148-161. doi:10.1016/S1474-4422(10)70322-7

22. Groppa S, Herzog J, Falk D, Riedel C, Deuschl G, Volkmann J (2014) Physiological and anatomical decomposition of subthalamic neurostimulation effects in essential tremor. Brain 137 (Pt 1):109-121. doi:10.1093/brain/awt304

23. Reich MM, Brumberg J, Pozzi NG, Marotta G, Roothans J, Astrom M, Musacchio T, Lopiano L, Lanotte M, Lehrke R, Buck AK, Volkmann J, Isaias IU (2016) Progressive gait ataxia following deep brain stimulation for essential tremor: adverse effect or lack of efficacy? Brain 139 (11):2948-2956. doi:10.1093/brain/aww223

24. Bouthour W, Wegrzyk J, Momjian S, Peron J, Fleury V, Tomkova Chaoui E, Horvath J, Boex C, Luscher C, Burkhard PR, Krack P, Zacharia A (2018) Short pulse width in subthalamic stimulation in Parkinson's disease: a randomized, double-blind study. Mov Disord 33 (1):169-173. doi:10.1002/mds.27265

25. Dayal V, Limousin P, Foltynie T (2017) Subthalamic Nucleus Deep Brain Stimulation in Parkinson's Disease: The Effect of Varying Stimulation Parameters. J Parkinsons Dis 7 (2):235-245. doi:10.3233/JPD-171077

26. Kroneberg D, Ewert S, Meyer AC, Kuhn AA (2019) Shorter pulse width reduces gait disturbances following deep brain stimulation for essential tremor. J Neurol Neurosurg Psychiatry 90 (9):1046-1050. doi:10.1136/jnnp-2018-319427

27. Anderson CJ, Anderson DN, Pulst SM, Butson CR, Dorval AD (2020) Neural selectivity, efficiency, and dose equivalence in deep brain stimulation through pulse width tuning and segmented electrodes. Brain Stimul 13 (4):1040-1050. doi:10.1016/j.brs.2020.03.017

28. Contarino MF, Bour LJ, Verhagen R, Lourens MA, de Bie RM, van den Munckhof P, Schuurman PR (2014) Directional steering: A novel approach to deep brain stimulation. Neurology 83 (13):1163-1169. doi:10.1212/WNL.0000000000000823 
29. Pollo C, Kaelin-Lang A, Oertel MF, Stieglitz L, Taub E, Fuhr P, Lozano AM, Raabe A, Schupbach M (2014) Directional deep brain stimulation: an intraoperative double-blind pilot study. Brain 137 (Pt 7):20152026. doi:10.1093/brain/awu102

30. Steigerwald F, Muller L, Johannes S, Matthies C, Volkmann J (2016) Directional deep brain stimulation of the subthalamic nucleus: A pilot study using a novel neurostimulation device. Mov Disord 31 (8):1240-1243. doi:10.1002/mds.26669

31. Dembek TA, Reker P, Visser-Vandewalle V, Wirths J, Treuer H, Klehr M, Roediger J, Dafsari HS, Barbe MT, Timmermann L (2017) Directional DBS increases side-effect thresholds-A prospective, double-blind trial. Mov Disord 32 (10):1380-1388. doi:10.1002/mds.27093

32. Rebelo P, Green AL, Aziz TZ, Kent A, Schafer D, Venkatesan L, Cheeran B (2018) Thalamic Directional Deep Brain Stimulation for tremor: Spend less, get more. Brain Stimul 11 (3):600-606. doi:10.1016/j.brs.2017.12.015

33. Helmich RC, Janssen MJ, Oyen WJ, Bloem BR, Toni I (2011) Pallidal dysfunction drives a cerebellothalamic circuit into Parkinson tremor. Ann Neurol 69 (2):269-281. doi:10.1002/ana.22361

34. Mure H, Hirano S, Tang CC, Isaias IU, Antonini A, Ma Y, Dhawan V, Eidelberg D (2011) Parkinson's disease tremor-related metabolic network: characterization, progression, and treatment effects. Neuroimage 54 (2):1244-1253. doi:10.1016/j.neuroimage.2010.09.028

35. Louis ED, Faust PL (2020) Essential tremor: the most common form of cerebellar degeneration? Cerebellum Ataxias 7:12. doi:10.1186/s40673-020-00121-1

36. Louis ED, Faust PL (2020) Essential tremor pathology: neurodegeneration and reorganization of neuronal connections. Nat Rev Neurol 16 (2):69-83. doi:10.1038/s41582-019-0302-1

\section{Tables}

Table 1: Demographic data and stimulation parameters of PD and ET patients 


\begin{tabular}{|c|c|c|c|c|c|c|c|c|c|c|}
\hline \multirow[t]{2}{*}{ Patient } & \multirow[t]{2}{*}{ Sex } & \multirow[t]{2}{*}{$\begin{array}{l}\text { Age } \\
\text { (years) }\end{array}$} & \multirow[t]{2}{*}{$\begin{array}{l}\text { Disease } \\
\text { duration } \\
\text { (years) }\end{array}$} & \multirow[t]{2}{*}{$\begin{array}{l}\text { Time } \\
\text { after } \\
\text { surgery } \\
\text { (weeks) }\end{array}$} & \multirow[t]{2}{*}{$\begin{array}{l}\text { Stimulation } \\
\text { side }\end{array}$} & \multicolumn{3}{|c|}{$\begin{array}{l}\text { Stereotactic } \\
\text { coordinates (mm) } \\
\text { lowest contact }\end{array}$} & \multicolumn{2}{|c|}{$\begin{array}{l}\text { Stimulation } \\
\text { amplitude } \\
(\mathrm{mA}) \\
\text { lowest / 2nd } \\
\text { lowest } \\
\text { contact }\end{array}$} \\
\hline & & & & & & $x$ & $y$ & z & $60 \mu \mathrm{s}$ & $30 \mu \mathrm{s}$ \\
\hline \multicolumn{11}{|c|}{ PD-patients } \\
\hline PD-1 & $M$ & 77 & 5 & 15 & Right & 9.69 & 8.73 & -5.4 & $\begin{array}{l}3 / \\
3.5\end{array}$ & $\begin{array}{l}4.21 \\
5\end{array}$ \\
\hline PD-2 & $M$ & 50 & 11 & 15 & Right & 11.87 & 7.96 & -1.87 & $\begin{array}{l}2.5 / \\
4.5\end{array}$ & $\begin{array}{l}3.5 / \\
6.4\end{array}$ \\
\hline PD-3 & $M$ & 74 & 4 & 46 & Right & 11.91 & 6.76 & -2.81 & $\begin{array}{l}2.8 / \\
3.3\end{array}$ & $\begin{array}{l}3.9 / \\
4.7\end{array}$ \\
\hline PD-4 & $M$ & 73 & 7 & 43 & Left & 13.28 & 8.54 & -1.56 & $3 /-$ & $4.2 /$ \\
\hline \multirow[t]{2}{*}{ PD-5 } & $M$ & 78 & 7 & 17 & Left & 10.99 & 9.69 & -5.17 & $\begin{array}{l}3.8 / \\
4.3\end{array}$ & $\begin{array}{l}5.4 / \\
6.1\end{array}$ \\
\hline & & & & & Right & 9.76 & 9.38 & -5.43 & $\begin{array}{l}3.1 / \\
3.5\end{array}$ & $\begin{array}{l}4.4 / \\
4.9\end{array}$ \\
\hline PD-6 & $M$ & 73 & 4 & 16 & Left & 10.82 & 6.65 & -2.97 & $\begin{array}{l}4.5 / \\
-\end{array}$ & $\begin{array}{l}6.4 / \\
-\end{array}$ \\
\hline \multicolumn{11}{|c|}{ ET-patients } \\
\hline \multirow[t]{2}{*}{ ET-1 } & $\mathrm{F}$ & 77 & 17 & 25 & Left & 11.82 & 9.4 & -2.54 & $\begin{array}{l}3.5 / \\
3\end{array}$ & $\begin{array}{l}4.95 \\
/ 4.2\end{array}$ \\
\hline & & & & & Right & 11.03 & 5.42 & -4.65 & $\begin{array}{l}2.5 / \\
3\end{array}$ & $\begin{array}{l}3.5 / \\
4.2\end{array}$ \\
\hline \multirow[t]{2}{*}{ ET-2 } & $M$ & 74 & 60 & 18 & Left & 11.79 & 8.93 & -4.75 & $\begin{array}{l}4 / \\
3.5\end{array}$ & $\begin{array}{l}5.7 / \\
5\end{array}$ \\
\hline & & & & & Right & 12.06 & 9.61 & -4.27 & $\begin{array}{l}3.5 / \\
3.5\end{array}$ & $5 / 5$ \\
\hline \multirow[t]{2}{*}{ ET-3 } & $M$ & 58 & 12 & 15 & Left & 10.34 & 8.49 & -3.75 & $\begin{array}{l}3 / \\
3.4\end{array}$ & $\begin{array}{l}4.21 \\
4.8\end{array}$ \\
\hline & & & & & Right & 8.62 & 8.14 & -4.41 & $\begin{array}{l}2.2 / \\
2.7\end{array}$ & $\begin{array}{l}3.1 / \\
3.8\end{array}$ \\
\hline ET-4 & M & 72 & 55 & 34 & Left & 11.4 & 7.03 & -4.76 & $3 /-$ & $4.2 /$ \\
\hline
\end{tabular}




\begin{tabular}{|c|c|c|c|c|c|c|c|c|c|c|}
\hline \multirow[t]{3}{*}{ Patient } & \multirow[t]{3}{*}{ Sex } & \multirow[t]{3}{*}{$\begin{array}{l}\text { Age } \\
\text { (years) }\end{array}$} & \multirow[t]{3}{*}{$\begin{array}{l}\text { Disease } \\
\text { duration } \\
\text { (years) }\end{array}$} & \multirow[t]{3}{*}{$\begin{array}{l}\text { Time } \\
\text { after } \\
\text { surgery } \\
\text { (weeks) }\end{array}$} & \multirow[t]{2}{*}{$\begin{array}{l}\text { Stimulation } \\
\text { side }\end{array}$} & \multicolumn{3}{|c|}{$\begin{array}{l}\text { Stereotactic } \\
\text { coordinates (mm) } \\
\text { lowest contact }\end{array}$} & \multicolumn{2}{|c|}{$\begin{array}{l}\text { Stimulation } \\
\text { amplitude } \\
(\mathrm{mA}) \\
\text { lowest / 2nd } \\
\text { lowest } \\
\text { contact }\end{array}$} \\
\hline & & & & & & $x$ & $y$ & z & $60 \mu \mathrm{s}$ & $30 \mu \mathrm{s}$ \\
\hline & & & & & Right & 10.67 & 8.25 & -3.81 & $\begin{array}{l}2.5 / \\
4\end{array}$ & $\begin{array}{l}3.5 / \\
5.7\end{array}$ \\
\hline \multirow[t]{2}{*}{ ET-5 } & $M$ & 53 & 20 & 15 & Left & 12.18 & 8.52 & -3.52 & $3.5 /$ & $4.9 /$ \\
\hline & & & & & Right & 8.68 & 6.28 & -6.05 & $\begin{array}{l}3 / \\
3.5\end{array}$ & $\begin{array}{l}4.2 / \\
4.9\end{array}$ \\
\hline \multirow[t]{2}{*}{ ET-6 } & $M$ & 54 & 35 & 21 & Left & 9.71 & 9.21 & -5.73 & $\begin{array}{l}2.9 / \\
3.5\end{array}$ & $\begin{array}{l}4.1 / \\
4.9\end{array}$ \\
\hline & & & & & Right & 9.14 & 9.75 & -5.64 & $\begin{array}{l}2.5 / \\
3\end{array}$ & $\begin{array}{l}3.5 / \\
4.2\end{array}$ \\
\hline \multirow[t]{2}{*}{ ET-7 } & $M$ & 63 & 36 & 26 & Left & 11.6 & 8.01 & -2.99 & $\begin{array}{l}2.8 / \\
3\end{array}$ & $\begin{array}{l}4 / \\
4.2\end{array}$ \\
\hline & & & & & Right & 10.35 & 6.9 & -1.53 & $\begin{array}{l}3.5 / \\
4.5\end{array}$ & $\begin{array}{l}4.9 / \\
6.4\end{array}$ \\
\hline
\end{tabular}

"Disease duration (years)" is calculated from symptom onset to the study assessment. "Time after surgery (weeks)" is calculated from the date of surgery to the study assessment. "Stimulation side" is the side of the electrode. "Stereotactic coordinates" are the coordinates of the lowest contact with $\mathrm{x}=$ lateral to midline, $\mathrm{y}=$ posterior to mid-commissural point (MCP), $\mathrm{z}$ = below the plane between anterior and posterior commissure, Stimulation amplitude is given of the of the lowest/ and $2^{\text {nd }}$ lowest contacts

Table 2: Patient characteristics of PD and ET patients at the group level 


\begin{tabular}{|c|c|c|c|c|}
\hline & & PD & ET & $\mathbf{p}$ \\
\hline \multicolumn{2}{|l|}{ Age (years) } & $70.8( \pm 10.42)$ & $64.43( \pm 9.91)$ & 0.28 \\
\hline \multicolumn{2}{|l|}{ Disease duration (years) } & $6.33( \pm 2,66)$ & $35.57( \pm 18.66)$ & 0.0047 \\
\hline \multicolumn{2}{|l|}{ Time after surgery (days) } & $174.8( \pm 102.68)$ & $162.57( \pm 52.55)$ & 0.79 \\
\hline \multicolumn{2}{|c|}{ Interval pre-post-DBS examination (days) } & $455.83( \pm 204.32)$ & $344.71( \pm 129.27)$ & 0.26 \\
\hline \multirow{3}{*}{$\begin{array}{l}\text { Stereotactic coordinates }(\mathrm{mm}) \\
\text { Lowest contact }\end{array}$} & $x$ & $11.25( \pm 1.27)$ & $10.65( \pm 1.25)$ & 0.35 \\
\hline & $\mathrm{y}$ & $8.51( \pm 0.96)$ & $8.14( \pm 1.30)$ & 0.55 \\
\hline & $\mathrm{z}$ & $-3.71( \pm 1.67)$ & $-4.17( \pm 1.27)$ & 0.52 \\
\hline \multirow{3}{*}{$\begin{array}{l}\text { Stereotactic coordinates }(\mathrm{mm}) \\
\text { 2nd lowest contact }\end{array}$} & $x$ & $11.92( \pm 1.07)$ & $11.56( \pm 1.17)$ & 0.57 \\
\hline & y & $7.01( \pm 0.88)$ & $6.65( \pm 1.26)$ & 0.58 \\
\hline & z & $-1.91( \pm 1.33)$ & $-2.09( \pm 1.27)$ & 0.80 \\
\hline \multicolumn{2}{|l|}{$\begin{array}{l}\text { Stimulation amplitude }(\mathrm{mA}) \\
\text { with } 60 \mu \mathrm{s}\end{array}$} & $3.58( \pm 0.68)$ & $3.19( \pm 0.53)$ & 0.06 \\
\hline
\end{tabular}

"Disease duration (years)" is calculated from symptom onset to the study assessment. "Time after surgery (days)" is calculated from the date of surgery to the study assessment. "Interval pre-post-DBS examination (days)" is calculated from the date of the pre-operative evaluation to the study assessment, "Stereotactic coordinates" are the coordinates of the lowest and 2 nd lowest contacts with $x=$ lateral to midline, $y=$ posterior to mid-commissural point (MCP), $\mathrm{z}=$ above the plane between anterior and posterior commissure, "Stimulation amplitude ( $\mathrm{mA})$ " is the suprathreshold amplitude to induce ataxia with $60 \mu$ s pulse width in ring mode

\section{Figures}






B

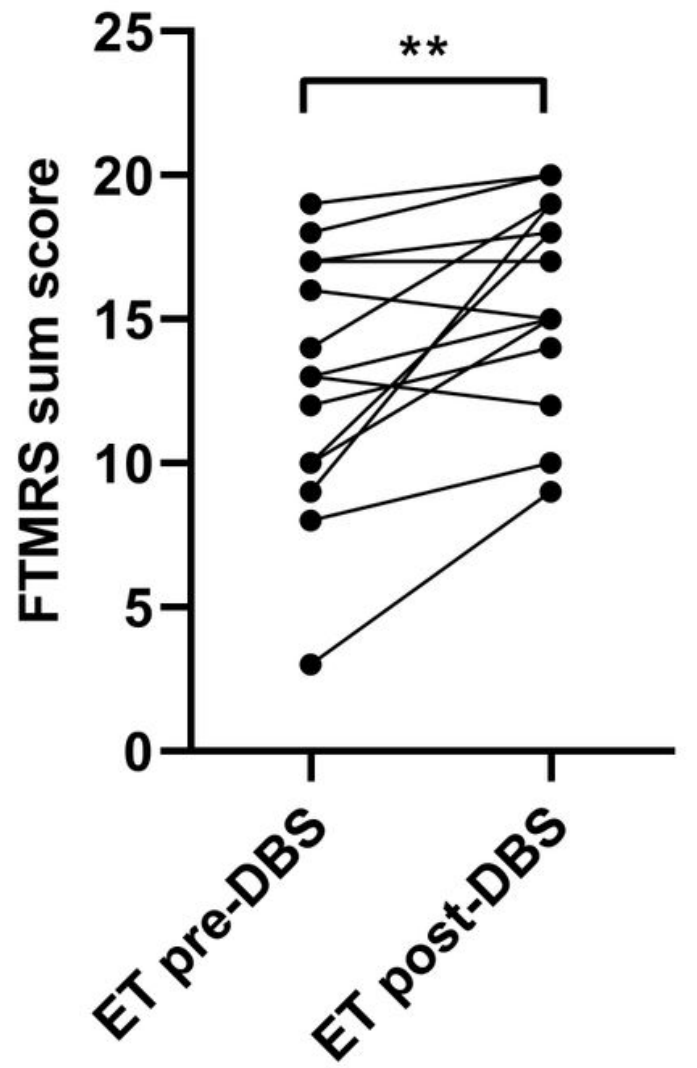

Figure 1

Pre- and Post-DBS tremor scores in PD and ET patients. A) PD patients were examined preoperatively in Med ON and Med OFF. For comparison, the postoperative examination Med ON Stim OFF is plotted. B) ET patients were examined pre- and postoperatively in a Med OFF condition. $\left({ }^{\star *} \mathrm{p}<0.01\right)$. 

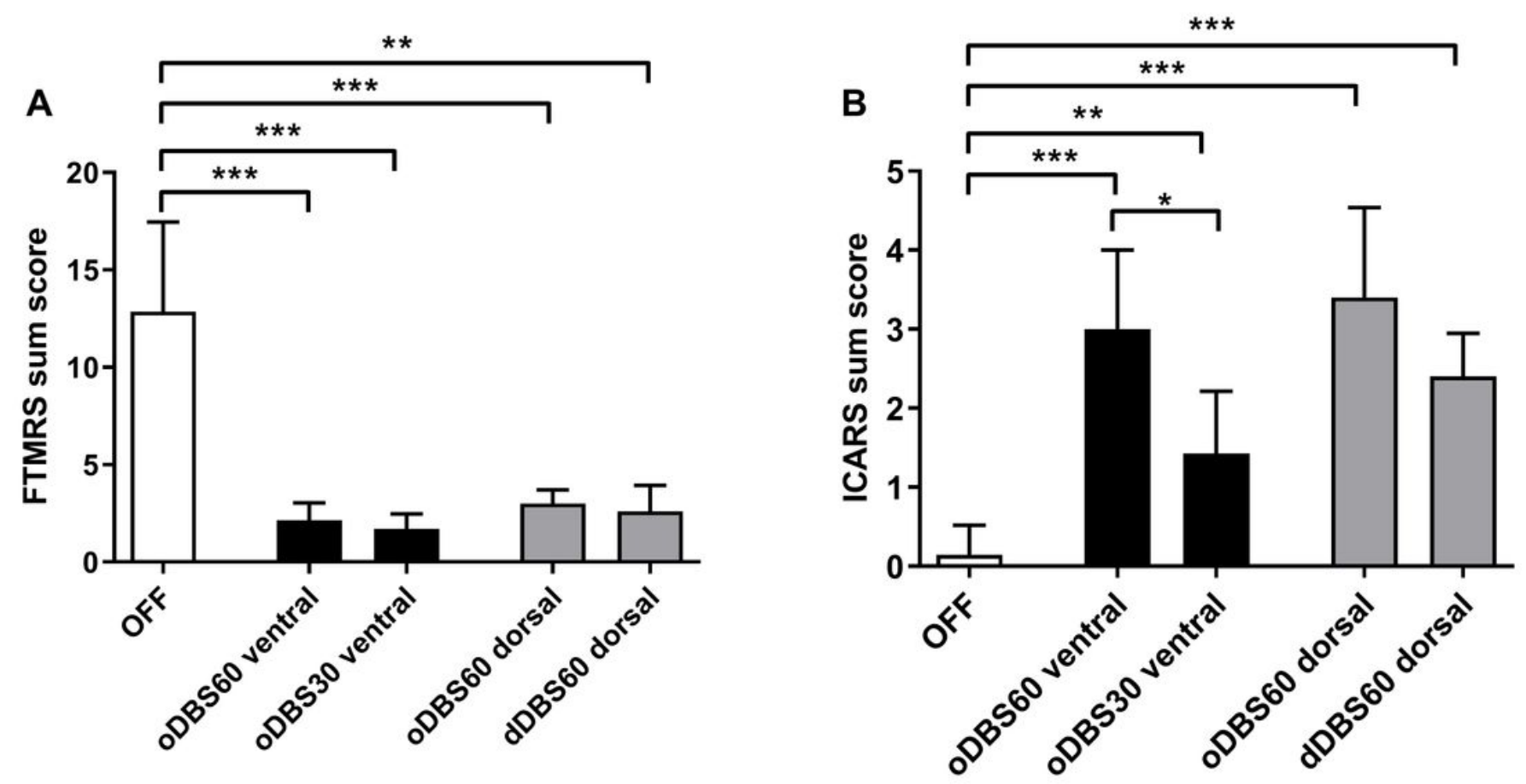

Figure 2

Tremor and ataxia scores in PD patients. The short pulse condition was studied on the most ventral contact, the segmented stimulation on the second lowest contact, so that the $60 \mu$ s ring stimulation is plotted on two heights (oDBS60 ventral and oDBS60 dorsal). A) All stimulation modes resulted in significant tremor reduction compared to Stim OFF with no difference between conditions. B) All stimulation conditions resulted in significant ataxia induction compared to Stim OFF. With short pulse stimulation, ataxia was significantly less pronounced than with $60 \mu$ s pulse. The lower ataxia score with directional stimulation compared to omnidirectional stimulation was not statistically significant with a small group size $(n=5)$ $\left({ }^{\star} p<0.05,{ }^{\star *} p<0.01,{ }^{\star \star \star} p \leq 0.001\right)$. 

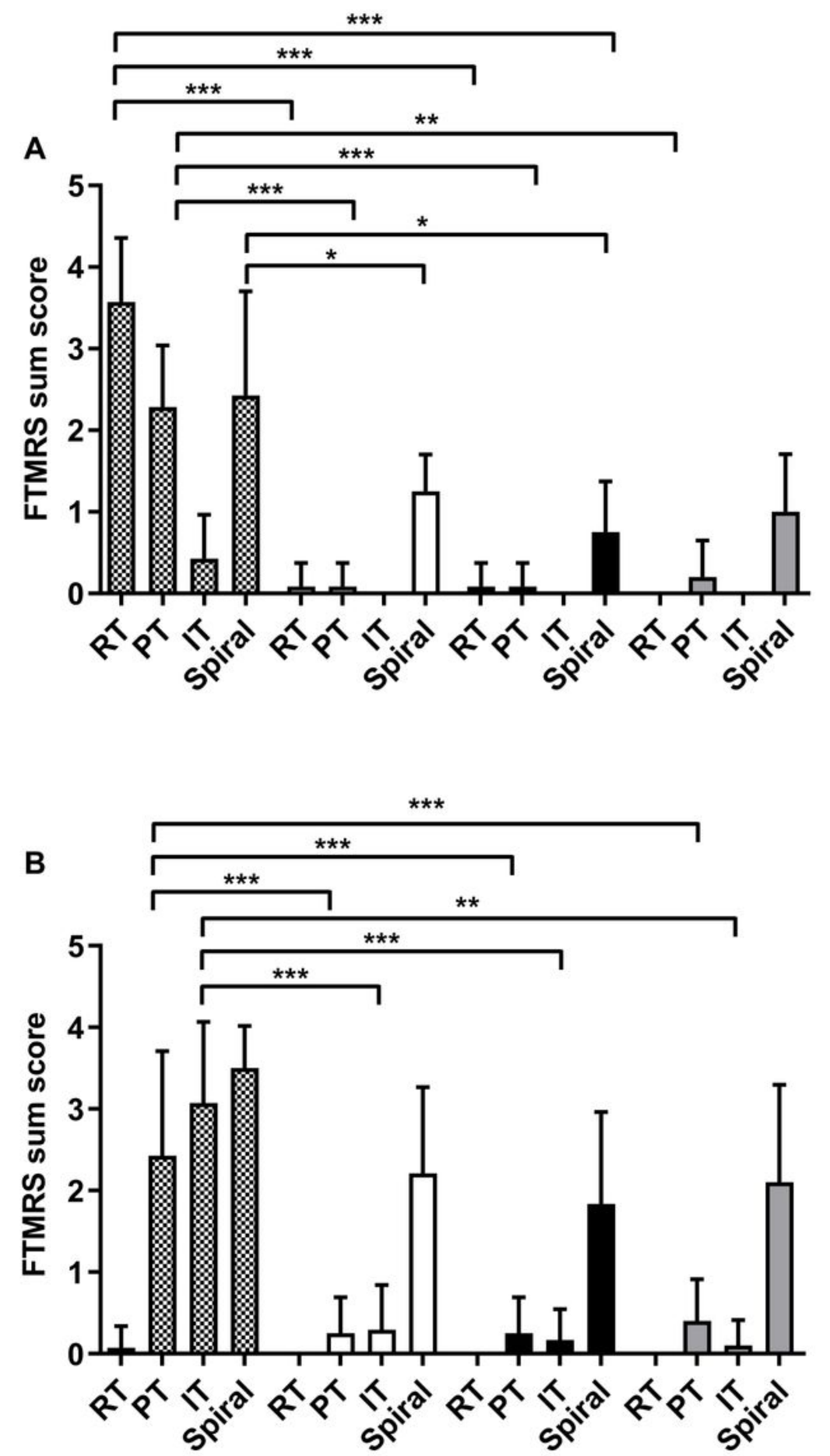

Figure 3

Response of tremor subtypes to the different stimulation conditions in PD (Fig 3A) and ET (Fig 3B). RT = rest tremor, $\mathrm{PT}=$ postural tremor, IT = intention tremor, Spiral = small spiral (item \#12 of the FTMRS) $(\star \star \star p<0.001)$. 

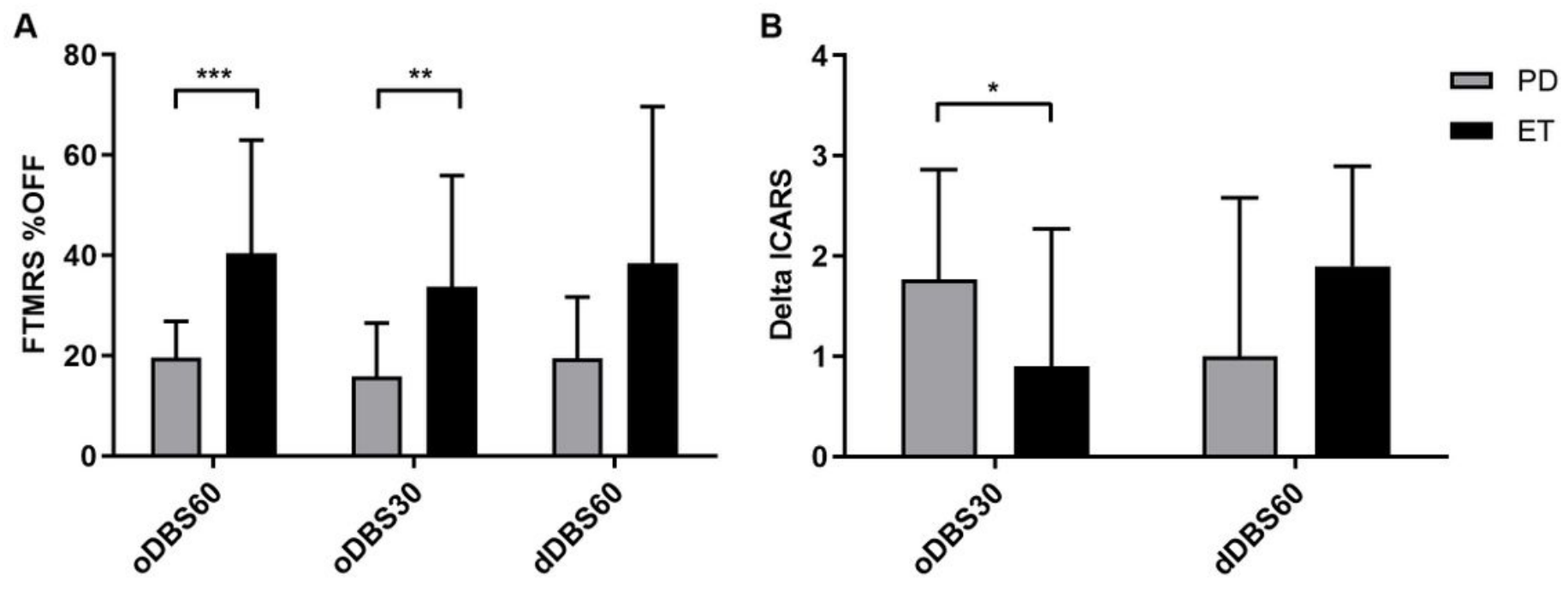

Figure 4

Differential effect of oDBS30 and dDBS60 on tremor (A) and ataxia (B) in PD and ET patients. A) Tremor score was normalized to the OFF FTMS-Score. Both oDBS60 and oDBS30 reduced tremor in PD more than in ET patients. B) Shown is the difference between the ataxia sum score at oDBS60 and the examined condition. In PD patients, oDBS30 reduced ataxia more than in ET patients. There was no significant difference for dDBS60. $\left({ }^{\star} p<0.05,{ }^{*} p<0.01,{ }^{* *} \mathrm{p} p<0.001\right)$ 